\title{
Zur Kenntnis des Eserins
}

\author{
Von \\ J. Herzig, k. M. K. Akad., und Hans Lieb \\ (Vorgelegt in der Sitzung am 10. Jänner 1918)
}

Das Eserin oder Physostigmin ist schon oft Gegenstand von Untersuchungen gewesen, ohne daß bis jetzt die Ermittlung der Konstitution desselben vollkommen gelungen wäré. Die Art der Bindung eines Stickstoffatoms ist bereits von Ehrenberg1 aufgeklärt worden. Es tritt bei der Einwirkung von Alkalien unter Wasseraufnahme als Methylamin gleichzeitig mit $\mathrm{CO}_{2}$ aus nach der Gleichung

$$
\underset{\text { Eserin }}{\mathrm{C}_{15} \mathrm{H}_{21} \mathrm{~N}_{3} \mathrm{O}_{2}}+\mathrm{H}_{2} \mathrm{O}=\mathrm{NH}_{2} \mathrm{CH}_{3}+\mathrm{CO}_{2}+\underset{13}{\mathrm{C}_{13} \mathrm{H}_{18} \mathrm{~N}_{2} \mathrm{O}} \text { Eserolin }
$$

Im Eserolin fand $\mathrm{Salway}{ }^{2}$ ein $\mathrm{CH}_{3}$ an $\mathrm{N}$ gebunden, was mit einer Angabe von Herzig und Meyer ${ }^{3}$ übereinstimmen würde, wonach im Physostigmin selbst zwei $\mathrm{CH}_{3}$-Gruppen nachweisbar sind. Spätere Untersuchungen von Straus ${ }^{4}$ ergaben, daß das Eserolinjodmethylat sich noch weiter abbauen läßt im Sinne der Gleichung

$$
\mathrm{C}_{13} \mathrm{H}_{18} \mathrm{~N}_{2} \mathrm{OCH}_{3} \mathrm{~J}=\mathrm{C}_{10} \mathrm{H}_{11} \mathrm{NO}+\mathrm{NH}\left(\mathrm{CH}_{3}\right)_{2} \mathrm{HJ}+\mathrm{C}_{2} \mathrm{H}_{2} \text {. }
$$

Was die Abspaltung der Gruppe $\mathrm{C}_{2} \mathrm{H}_{2}$ betrifft, so ist noch keine genügende Klarheit vorhanden, aber die Frage kommt

1 Verh. d. Naturf. u. Ärzte, 1893, p. 102.

2 Journ. Chem. Soc., 101, (1) 978 (1912).

3 Monatshefte für Chemie, 18, 289 (1897).

4 Ann. d. Chem., 401, 350 (1914), 406, 332 (1910). 
hier nicht in Betracht. Von Wichtigkeit ist für uns, daß in dem Zersetzungsprodukt $\mathrm{C}_{10} \mathrm{H}_{11} \mathrm{NO}$, von Straus Physostigmol genannt, nach den Bestimmungen von Lieb noch immer sine Methylimidgruppe nachgewiesen werden kann. Dieser Nachweis steht im Widerspruch zu den Bestimmungen von Herzig und Meyer beim Eserin selbst. Wenn nämlich im Physostigmol noch eine Methylimidgruppe vorhanden wäre, so müßten das Eserolin deren zwei und das Eserin deren drei enthalten.

Die Methylimidbestimmung von Lieb beim Physostigmol war nach der von Pregl und Lieb ${ }^{1}$ ausgearbeiteten Mikromethylimidmethode ausgeführt worden, welche im Prinzip der Makromethode von Herzig und Meyer nachgebildet ist. Es liegt also der Fall vor, daß nach der Makromethode beim Eserin eine Methylimidgruppe zu wenig gefunden worden wäre. Tatsächlich hat dann Straus ${ }^{2}$ beim Eserolin nach der Mikromethode fast genau den doppelten Wert erhalten als nach der Makromethode. Die Zahlen mögen hier folgen:

Ber. fiur $1 \mathrm{CH}_{3} 6 \cdot 88 \% \mathrm{CH}_{3}$.

Gef. Makro I. $7 \cdot 650 / 0 \mathrm{CH}_{3}$, II. $7 \cdot 66 \% / 0 \mathrm{CH}_{3}$.

Gef. Mikro I. $13 \cdot 300 \% \mathrm{CH}_{3}$, II. $11 \cdot 940 \mathrm{CH}_{3}$.

Diese auffallende Erscheinung hat uns veranlaßt, der Sache näher zu treten und zu untersuchen, wie sich die Verhältnisse beim Eserin selbst sowie beim salicylsauren Eserin gestalten werden. Es hat nun vorerst Lieb beide Verbindungen nach der Mikromethode untersucht und die von ihm gefundenen Zahlen sprechen unzweifelhaft dafür, daß tatsächlich nach dieser Methode drei $\mathrm{CH}_{3}$-Gruppen am Stickstoff nachgewiesen werden können. Dabei sollte auch untersucht werden, ob und welche Rolle der Zusatz von Jodammonium spielt.

Diese Bestimmungen führten mit und ohne Zusatz von Jodammonium zu folgenden Zahlen:

1 Preg1, Quantitative organ. Mikiroanalyse, p. 162 (Verlag J. Springer, Berlin).

3 Ann. d. Chem., 406, 332 (1915). 


\begin{tabular}{|c|c|c|}
\hline \multicolumn{2}{|c|}{ Eserin } & \multirow{2}{*}{$\begin{array}{l}\text { Salicylsaures Eserin } \\
\text { mit } \mathrm{NH}_{4} \mathrm{~J}\end{array}$} \\
\hline mit $\mathrm{NH}_{4} \mathrm{~J}$ & olnne $N H_{4} \mathbf{J}$ & \\
\hline $\begin{array}{l}\text { I. } 14 \cdot 700 \% \mathrm{CH}_{3} \\
\text { II. } 16 \cdot 15\end{array}$ & $\begin{array}{l}\text { I. } 16 \cdot 68 \% \mathrm{CH}_{3} \\
\text { II. } 14 \cdot 71\end{array}$ & $\begin{array}{l}\text { I. } 10 \cdot 62 \% \mathrm{CH}_{3} \\
\text { II. } 11 \cdot 19\end{array}$ \\
\hline III. $16 \cdot 34$ & III. $13 \cdot 76$ & \\
\hline
\end{tabular}

Der Zusatz von Jodammonium hat also auf das Resultat keinen Einfluß. Rechnet man diese gefundenen Werte, welche Gewichte von $\mathrm{CH}_{3}$ in Prozenten der angewendeten Substanz angeben, auf die im Molekül am Stickstoff enthaltene Anzahl von $\mathrm{CH}_{3}$-Gruppen um, so ergeben sich für das

\begin{tabular}{|c|c|c|}
\hline \multicolumn{2}{|c|}{ Eserin } & Salicylsaure Eserin \\
\hline mit $\mathrm{NH}_{4} \mathrm{~J}$ & ohne $\mathrm{NH}_{4} \mathrm{~J}$ & mit $\mathrm{NH}_{4} \mathrm{~J}$ \\
\hline $2 \cdot 72-3 \cdot 02$ & $2 \cdot 54-3 \cdot 08$ & $2 \cdot 87-2 \cdot 92$ \\
\hline
\end{tabular}

Nachdem diese Tatsache festzestellt war, sind wir zur Untersuchung des Eserins nach der Methode von Herzig und Meyer geschritten. Zwischen Mikro- und Makrobestimmung kam auf den ersten Blick als Hauptunterschied der in Betracht, daß bei der Mikrobestimmung infolge der kleineren Apparatur eine höhere Temperatur erzielt werden könnte. Bei der Wiederholung der Makrobestimmung ging daher unser Bestreben dahin, die Funktion der Temperatur genau zu studieren. Es sind infolgedessen die Bestimmungen derart vorgenommen worden, daß bei der ersten Destillation die Temperatur ungefähr bei $310^{\circ} \mathrm{C}$. gehalten, während sie bei der zweiten und dritten Destillation bis zu $360^{\circ} \mathrm{C}$. gesteigert wurde. Die erhaltenen Daten waren folgende: ${ }^{1}$

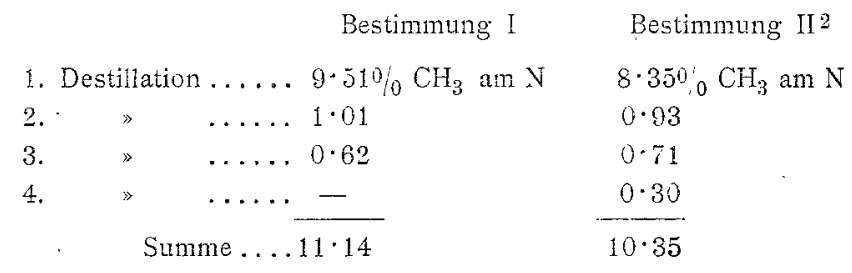

1 Alle Malsrobestimmungen sind von Frl. M. Quittner ausgeführt worden.

2 Derlei Bestimnungen mit drei und mehr Wiederhoiungen sind jetzt ganz leicht ausfihrbar, seitdem Doppelkölbchen aus Quarz in Anwendung 
Es berechnen sich fïr

$$
\begin{array}{cr}
\text { Eserin mit } 2 \mathrm{CH}_{3} & \text { Eserin mit } 3 \mathrm{CH}_{3} \\
10.90 / 0 \mathrm{CH}_{3} & 16.35 \% \\
\mathrm{CH}_{3}
\end{array}
$$

Es sind demnach nach der Makromethode einwandfrei nur zwei Methylgruppen nachgewiesen worden, wobei außerdem die Temperatur jedenfalls höher war als bei der von Herzig und Meyer seinerzeit publizierten Bestimmung, welche den Wert von $8 \cdot 8 \% \mathrm{CH}_{3}$ am $\mathrm{N}$ ergeben hatte.

Ein zweiter Umstand, der von Einfluß sein konnte, war das Verhältnis der Substanzmenge zu der Menge der hinzugefügten Jodwasserstoffsäure. Während nämlich nach der gewöhnlichen Methode von Herzig und Meyer auf ungefähr 0.3 bis $0.4 \mathrm{~g}$ Substanz in der Regel $10 \mathrm{~cm}^{3} \mathrm{HJ}$ angewandt werden, sind nach der Angabe von Pregl und Lieb für je 3 bis $4 \mathrm{mg}$ Substanz zirka $2 \mathrm{~cm}^{3}$ Jodwasserstoff hinzugefügt worden. Die Anwendung eines so großen Überschusses von Jodwasserstoff ist bei der Apparatur der Makrobestimmung ausgeschlossen, die gebräuchliche Menge kann höchstens verdoppelt werden. Man kann sich aber den Verhältnissen bei der Mikromethode dadurch noch mehr nähern, daß man anstatt 0.3 bis $0.4 g$ nur $0.1 \mathrm{~g}$ Substanz in Anwendung bringt. Bestimmungen, die von diesem Gesichtspunkt ausgehend unternommen wurden, lieferten tatsächlich folgende Resultate:
I. $15 \cdot 340 \% \mathrm{CH}_{3}$,
II. $15.09 \% \mathrm{CH}_{3}$,
III. $16 \cdot 130_{: 0}^{:} \mathrm{CH}_{3}$.

Die Substanzmengen waren bei
I. $0.097 \mathrm{~s}$,
II. $0 \cdot 08548$
III. $0.080 \mathrm{~g}$.

Von der Jodwasserstoffsäure wurden in allen diesen Fällen zirka $20 \mathrm{~cm}^{3}$ angewendet. Aus den Zahlen ist zu schließen, daß tatsächlich die Menge der Jodwasserstoffsäure

lsommen, welche sich, wie bereits in Hans Meyer, Analyse und Konstitutionsbestimmung usw., 3. Aufl., mitgeteilt, sehr bewähren. Es ist schwer, die Öffnungen der Kölbchen gleichmäßig rund herzustellen und das ergibt natüriich eine Schwierigleeit beim Einpassen der Korkstöpse1. Diese Schwierigkeit išt leicht zu umgehen, wenn man die Öfnungen innen scliwach konisch ausschleifen läßt. 
nicht gleichgültig ist. Es scheint aber auch die Temperatu: nicht unwesentlich $z \mathrm{u}$ sein. Während bei Versuch I und II die Temperatur schon bei der ersten Destillation ziemlich hoch war, wurde der Versuch III derart variiert, daß bei der ersten und zweiten Destillation die Temperatur unter $330^{\circ} \mathrm{C}$. blieb, während sie bei der dritten Destillation bis $380^{\circ} \mathrm{C}$. stieg. Der Verlauf der Reaktion ist aus folgender Zusammenstellung $\mathrm{Zu}$ ersehen:

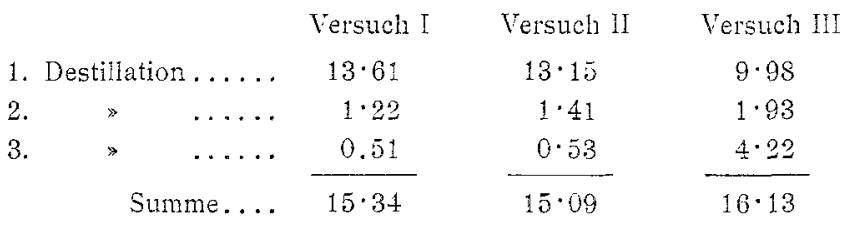

Da nun die theoretisch für drei Methylgruppen geforderte Zahl $16 \cdot 35 \% \mathrm{CH}_{3}$ beträgt, so ist jedenfalls durch diese Bestimmungen bewiesen, daß bei Anwendung von $20 \mathrm{~cm}^{3}$ Jodwasserstoffsäure und zirka $0.1 \mathrm{~g}$ Substanz auch nach der gewöhnlichen Makromethode bei höherer Temperatur die für drei Gruppen geforderten Werte erhalten werden können. Über die Rolle, die hier der größere Überschuß an Jodwasserstoffsäure spielt, sind verschiedene Annahmen möglich. Man könnte daran denken, daß am Stickstoff alkylierte Pyrrol- und Indoiderivate, welche nach den bisherigen Erfahrungen die substituierende Gruppe nicht quantitativ abspalten lassen, bei Anwendung eines Überschusses von Jodwasserstoffsäure sich ganz allgemein normal verhalten würden. Versuche, die in dieser Richtung ausgeführt wurden, zeigten, daß dies nicht der Fall. So ergab ein

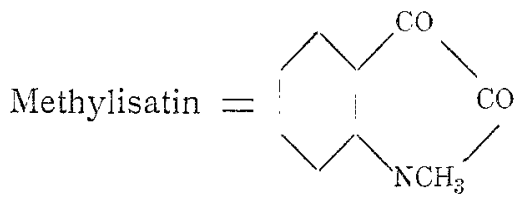

welches wir der Güte des Herrn Prof. Kohn verdanken, bei Anwendung von $0.315 \mathrm{~g}$ Substanz und $10 \mathrm{~cm}^{3}$ Jodwasserstoffsäure $4.20 \% \mathrm{CH}_{3}$, bei Anwendung von $0.103 \mathrm{~g}^{\circ}$ und $20 \mathrm{~cm}^{3}$ Jodwasserstoffsäure selbst bei $400^{\circ} \mathrm{C}$. nur $4.90 \% \mathrm{CH}_{3}$, während sich theoretisch $9 \cdot 32 \% \quad \mathrm{CH}_{3}$ berechnen. 
Eine zweite Substanz von der Formel

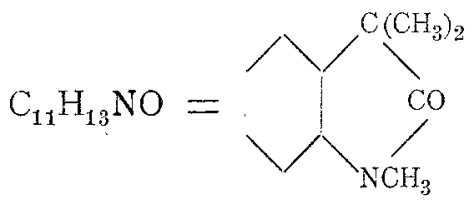

welche uns von Herrn Prof. Brunner aus Innsbruck gütigst zur Verfügung gestellt worden war, zeigte ein ganz atypisches Verhalten. Die Zersetzung ging erst über $300^{\circ} \mathrm{C}$. vor sich, war sehr stürmisch, ja geradezu explosionsartig, indem unter starkem Stoßen vollkommene Verharzung eintrat. Die erste Destillation konnte trotz der explosionsartigen Zersetzung zu Ende geführt werden. Die ausgeschiedene Menge Jodsilber ergab einen Wert von $0.97 \% \mathrm{CH}_{3}$, während theoretisch $8.58 \%$ gefordert wird. Auch nach der Mikroanalyse waren nach Bestimmungen von Lieb bei diesen beiden Substanzen keine brauchbaren Resultate zu erzielen. Demgegenüber stehen aber folgende Tatsachen: Lieb $^{1}$ selbst hat beim Physostigmol, welches nach Straus ein Pyrrolderivat sein muß, die Methylimidgruppe mikroanalytisch fast quantitativ nachgewiesen (gef. $6 \cdot 93 \% \mathrm{CH}_{3}$, ber. $8 \cdot 68 \%$ ). Ein noch besseres Resultat erzielte Straus ${ }^{1}$ beim Physostigmolmethyläther (gef. $8 \cdot 61 \%$, ber. $8 \cdot 58 \% \mathrm{CH}_{3}$ ).

Ein ähnlich gutes Resultat wird auch von Straus ${ }^{1}$ für das N-Methylindol angegeben (gef. $22 \cdot 14 \%$, ber. $22 \cdot 00 \%$ $\mathrm{CH}_{3}$ ).

Die beiden oben genannten Präparate, Physostigmol und $\mathrm{N}$-Methylindol, standen uns nicht zur Verfügung und so konnten die Versuche in keinerlei Richtung wiederholt werden.

Bei der momentanen Sachlage kann daher bis auf weiteres nur die Annahme gemacht werden, daß einzelne Pyrrol- und Indolderivate durch Anwendung überschüssiger Jodwasserstoffsäure bei höherer Temperatur derartig umgewandelt werden, daß der Alkylrest nach der Methode von Herzig und Meyer doch quantitativ bestimmbar wird. Dabei ist natürlich bei dieser 
jedenfalls komplizierten Reaktion die Möglichkeit nicht ausgeschlossen, daß dieser Alkylrest erst bei der Umwandlung entsteht. In dieser Richtung wird hoffentlich die mit so schönem Erfolge begonnene Untersuchung von Straus noch Aufschluß bringen.

Es soll außerdem hier noch eine Tatsache erwähnt werden, welche möglicherweise für die Untersuchung des Physostigmins von heuristischem Wert sein könnte. Kirpal und Bühn ${ }^{1}$ haben in der letzten Zeit eine Methode zur Bestimmung der Methylgruppen zunächst nur für schwefelhaltige organische Substanzen ausgearbeitet, welche aber auch sonst gewisse Vorteile bietet. Die Methode beruht darauf, daß das Jodmethyl sich quantitativ an das Pyridin addiert, daher durch Pyridin quantitativ absorbiert werden kann. Nach dem Abdampien des Pyridins ist die Menge des Jodmethylats durch Titration bestimmbar. Die von Kirpal für Methoxyl verwendete Wethode ist dann später in dem hiesigen Laboratorium durch Zipperer bei der Methylimidbestimmung verwertet worden und es hat sich eine sehr gute Übereinstimmung der Werte nach der gewöhnlichen Methode (alkoholische Silbernitratlösung) mit denen nach der Pyridinmethode nachweisen lassen. Die Pyridinmethode gibt nun aber auch die Möglichkeit, mit einem gewissen Grade von Wahrscheinlichkeit die Natur des abgeschiedenen Jodalkyls zu beurteilen. Kirpal hat nämlich gezeigt, daß seine Methode bei Äthoxylderivaten nicht mehr anvendbar ist, weil das Jodäthyl sich nicht so quantitativ an das Pyridin addiert wie das Jodmethyl.

Es ist sehr wahrscheinlich, daß mit einzelnen Ausnahmen sich die Mehrzahl der höheren Alkyljodide ähnlich verhalten wird wie Jodäthyl. Während also bei der Abspaltung von Jodmethyl die Werte nach Herzig und Meyer und nach Kirpal übereinstimmen, müßte sich im allgemeinen bei anderen Alkylresten eine Differenz zwischen den beiden Werten ergeben. Mit Rücksicht auf dieses nicht unwichtige Moment hat dann Lieb ${ }^{2}$ auch die Methode von Kirpal mikroanalytisch

1 Berl. Ber., 4z, 1084 (1914) - Monatsh. f. Chem., 36, 853 (1915).

2 Preg1, Quantitative organische Mikroanalyse, p. 166. 
ausgearbeitet und das Eserin sowie salicylsaure Eserin mikroanalytisch nach Kirpal untersucht. Während sich nun, wie schon oben erwähnt, mikroanalytisch nach der gewöhnlichen Methode von Herzig und Meyer beim Eserin folgende Werte für die Anzahl der $\mathrm{CH}_{3}$-Gruppen im Molekül ergeben:

$$
\begin{array}{cl}
\text { Mit } \mathrm{NH}_{4} \mathrm{~J} & \text { Olne } \mathrm{NH}_{4} \mathrm{~J} \\
2.72-3.02 & 2.54-3.03
\end{array}
$$

liefern die von Lieb mikroanalitisch nach Kirpal gefundenen Zahlen $\left(12 \cdot 65,13 \cdot 16,12 \cdot 92,13 \cdot 86 \% \mathrm{CH}_{3}\right.$ am $\left.\mathrm{N}\right)$ für die Anzahl der Methylgruppen im Molekül folgende Grenzwerte:

$$
2 \cdot 34-2 \cdot 56 .
$$

Beim salicylsauren Eserin liegen die Verhältnisse ähnlich

$$
\begin{array}{cc}
\text { Herzig-Meyer } & \text { Kirpál } \\
2.87-2.92 & 2.32 .
\end{array}
$$

Angesichts dieser konstanten, jedenfalls bemerkenswerten Differenz fällt es schwer, an einen Zufall $z u$ denken. Die Möglichkeit, daß die dritte im Eserin abspaltbare Gruppe nichi auf eine präformierte oder erst entstehende Methyl-, sondem auf eine andere Alkylgruppe zu beziehen wäre, ist also nicht ausgeschlossen, zumal gegen die Annahme, daß beispielsweise eine Äthylgruppe vorliegt, beim jetzigen Stande unserer Kenntnisse kein Einwand erhoben werden kann und Straus selbst diese Möglichkeit vorläufig in Betracht zieht. Andrerseits ist die Verwendbarkeit der Kirpa1-Methode in dieser Richtung noch gar nicht erprobt und daher einige Vorsicht geboten. Die definitive Entscheidung muß auch in dieser Richtung den weiteren Studien von Straus vorbehalten bleiben. 